Sleep and Lipid Profile During Transition from Childhood to Adolescence

\author{
Kuula-Paavola, Liisa
}

2016

Kuula-Paavola , L , Pesonen , A-K, Kajantie , E, Lahti , J , Andersson , S , Strandberg , T \& Räikkönen, K 2016 , ' Sleep and Lipid Profile During Transition from Childhood to Adolescence ' , The Journal of Pediatrics , vol. 177 , pp. 173-178e . https://doi.org/10.1016/j.jpeds.2016.06.026

http://hdl.handle.net/10138/231306

https://doi.org/10.1016/j.jpeds.2016.06.026

publishedVersion

Downloaded from Helda, University of Helsinki institutional repository.

This is an electronic reprint of the original article.

This reprint may differ from the original in pagination and typographic detail.

Please cite the original version. 


\title{
Sleep and Lipid Profile During Transition from Childhood to Adolescence
}

\author{
Liisa Kuula, $\mathrm{MA}^{1}$, Anu-Katriina Pesonen, $\mathrm{PhD}^{1}$, Eero Kajantie, MD, $\mathrm{PhD}^{2,3,4}$, Jari Lahti, $\mathrm{PhD}^{1,5,6}$, Sture Andersson, MD, $\mathrm{PhD}^{2}$, \\ Timo Strandberg, MD, $\mathrm{PhD}^{7,8}$, and Katri Räikkönen, $\mathrm{PhD}^{1}$
}

Objectives To assess the longitudinal effects of sleep duration and quality on lipid profiles during the transition from childhood to early adolescence, over a 4-year-period.

Study design A cohort study of children born in 1998 examined at 8 years of age (SD, 0.3; $n=105)$ and 12 years of age $(S D, 0.5 ; n=190)$. Sleep duration, wake after sleep onset, sleep efficiency, and weekend catch-up sleep were measured with actigraphs for 7 ( 8 years of age) and 8 (12 years of age) nights. Fasting serum samples were collected at 12 years of age. Covariates included age, pubertal development, socioeconomic status, body mass index, and physical activity.

Results In girls, shorter sleep duration at 8 and 12 years of age was associated with lower high-density lipoproteincholesterol and higher triglycerides at 12 years of age. Poorer sleep quality at 8 years of age and longer weekend catch-up sleep at 12 years of age was associated with higher triglycerides at 12 years of age. From 8 to 12 years of age, improvement in sleep quality associated with higher total cholesterol, and a decrease in sleep duration with lower lipid levels. In boys, longer sleep duration at 8 years of age, and a larger decrease in sleep duration from 8 to 12 years of age was associated with higher levels of triglycerides at 12 years of age.

Conclusions Poorer sleep during transition to early adolescence is associated with an atherogenic lipid profile in early adolescent girls, and such effects are less prominent in boys. Poor sleep may have long-term associations with health, which are not mitigated by the amount of physical activity. (J Pediatr 2016;177:173-8).

leep and health are intertwined already in childhood. Short sleep and poor sleep quality are associated with overweight and obesity, ${ }^{1,2}$ increased mental health problems, ${ }^{3,4}$ and adverse neuroendocrine changes ${ }^{5}$ in children, and toward adolescence, risks related to cardiometabolic disease begin to emerge. ${ }^{6-9}$

To understand the mechanisms underlying the relationships between sleep and somatic health outcomes, more information is needed to clarify the developmental trajectories of these associations from childhood to adolescence. In particular, it is not yet well-known how sleep quantity and sleep quality over the transition from childhood to adolescence are associated with metabolic risk factors.

With regard to cross-sectional findings, one study reported that longer sleep duration as measured objectively with actigraphy had a beneficial effect on overall lipid metabolism in a community cohort of children aged 4-10 years, one-half of whom were overweight or obese. ${ }^{10}$ Shorter sleep duration, especially in the presence of irregular sleep patterns, was associated with higher plasma fasting insulin, low-density lipoprotein cholesterol (LDL-C), and C-reactive protein concentrations. Some studies including children and adolescents with more healthy body mass indices (BMI), have not found associations between objectively measured sleep and lipids. ${ }^{11,12}$ Conclusions of the existing cross-sectional studies are, however, unclear because many studies have not measured sleep objectively ${ }^{13-15}$ or include a large age variation between the participants. ${ }^{10}$

The few longitudinal studies have indicated that sleep patterns in childhood have far-reaching effects on other indicators of metabolic risks: one study reported that persistently short sleep duration during childhood increased the risk to become obese or overweight by up to 4.2 -fold, ${ }^{2}$ and another reported that children with continuously insufficient sleep duration from infancy to school age had higher metabolic risk scores. ${ }^{16}$ However, to our knowledge no previous study has reported the longitudinal impact of sleep on lipid metabolism using objectively measured sleep in this age group.

$\begin{array}{ll}\text { BMI } & \text { Body mass index } \\ \text { HDL-C } & \text { High-density lipoprotein cholesterol } \\ \text { WASO } & \text { Wake after sleep onset } \\ \text { LDL-C } & \text { Low-density lipoprotein cholesterol } \\ \text { TC } & \text { Total cholesterol } \\ \text { TGs } & \text { Triglycerides }\end{array}$

From the ${ }^{1}$ Institute of Behavioral Sciences, University of Helsinki, Finland; ${ }^{2}$ Children's Hospital, Helsinki University Hospital, University of Helsinki, Helsinki, Finland;

${ }^{3}$ Chronic Disease Prevention Unit, National Institute for Health and Welfare, Helsinki, Finland; ${ }^{4}$ Department of Obstetrics and Gynecology, MRC Oulu, Oulu University Hospital, University of Oulu, Oulu, Finland; ${ }^{5}$ Helsinki Collegium for Advanced Studies, University of Helsinki, Helsinki, Finland; ${ }^{6}$ Folkhälsan Research Centre, Helsinki, Finland; ${ }^{7}$ University of Helsinki, Helsinki University

Central Hospital, Helsinki, Finland; and ${ }^{8}$ Center for Life Course Epidemiology and Systems Medicine, University of Oulu, Oulu, Finland

Supported by Academy of Finland (1287174), PsyCo Doctoral Programme, Päivikki and Sakari Sohlberg Foundation, Finska Läkaresällskapet, University of Helsinki, Emil Aaltonen Foundation, Foundation for Pediatric Research, Juho Vainio Foundation, Novo Nordisk Foundation, Signe and Ane Gyllenberg Foundation, Sigrid Juselius Foundation, The Paulo Foundation, Helsinki University Central Hospital. The authors declare no conflicts of interest.

Portions of the study were presented at the Congress of the European Sleep Research Society, Bologna, Italy, September 13-16, 2016.

0022-3476/\$ - see front matter. @ 2016 Elsevier Inc. All rights reserved.

http://dx.doi.org10.1016/j.jpeds.2016.06.026 
We investigated the longitudinal effects of actigraphymeasured sleep duration and quality on lipid profiles during the transition from childhood to early adolescence over a 4-year period. Additionally, we examined the associations between irregular sleep patterns and lipid profiles; previous studies have reported that sleep irregularity increases risks related to health outcomes. ${ }^{10,17}$

\section{Methods}

Participants came from an urban community-based cohort composed of 1049 healthy singletons born between March and November 1998 in Helsinki, Finland. ${ }^{18}$ Details are described in previous reports. ${ }^{19,20}$ In 2006 (at 8 years of age), we invited a subsample of the initial cohort members who had given permission to be included in a follow-up and who were traceable ( $\mathrm{n}=413$ invited, $\mathrm{n}=321$ participated [77\%]; 164 girls and 157 boys; mean age, 8.1 years [SD, 0.3; range, 7.4-8.9]). This subsample was weighted on mothers who in pregnancy consumed more glycyrrhizin (which inhibits placental $11 \beta$ HSD2 function) in the form of licorice..$^{20}$ Of the 321 participants, 297 (92.5\%) took part in the sleep measurement, of whom 296 had valid sleep measurements on a minimum of 3 nights, and of them, valid fasting blood serum samples at 12 years of age were available from 105 participants (35\%). The Ethics Committees of the City of Helsinki Health Department and Children's Hospital in Helsinki University Central Hospital approved the study protocol. Each child and her or his parent(s) provided their written informed consent at both follow-up visits.

In 2009-2011 (at 12 years of age), all the initial cohort members $(\mathrm{n}=1049)$ who had given permission to be contacted and whose addresses were traceable $(n=920 ; 87.7 \%$ of the original cohort) were invited to a follow-up, of which 692 (75.2\%) could be contacted by phone (mothers of the adolescents). Of them, 451 (234 girls and 217 boys; $49 \%$ of the invited; $65.2 \%$ of the contacted) participated in a follow-up at a mean age of 12.3 years (SD, 0.5 ; range, 11.0-13.2). Of the 451 participants, $362(80 \%)$ took part in the sleep measurement, of whom $358(79 \%)$ had valid sleep measurement on a minimum of 3 nights. Of them, valid fasting blood serum samples were available from 190 participants (53\%).

Complete sleep actigraphy data from both the 8 and 12 years of age measurement points were available for 188 adolescents ( 99 girls, 89 boys; $64 \%$ of those with valid actigraphy data from 8 years of age), and of these, fasting serum sample data from 95 ( 50 girls, 45 boys). Thus, our analytical sample consisted of 190 in cross-sectional analyses at 12 years of age and 105 in longitudinal analyses associating sleep at 8 years of age and lipids at 12 years of age (note that 10 of these participants in the longitudinal sample did not have complete sleep data at both ages 8 and 12 years of age and lipids at 12 years of age, allowing the analyses of change in sleep).

The cross-sectional sample $(\mathrm{n}=190)$ did not differ (all $P \geq .06$ ) from the rest of the participants at 12 years of age in BMI, age, mother's BMI, mother's age at birth, gestation age, maternal alcohol consumption, length at birth, birthweight, physical activity, pubertal development, socioeconomic status, or maternal licorice consumption. With regard to the initial cohort, there were no differences (all $P \geq 0.11$ ) in mother's BMI, mother's age at birth, maternal licorice consumption, maternal alcohol consumption, gestation age, length at birth, and birthweight.

The longitudinal sample with sleep measurements and lipid profiles available did not differ (all $P \geq .09$ ) from the rest of the sample at 8 years of age in age, mother's age at birth, mother's BMI, gestation age, length at birth, birth weight, physical activity, and maternal licorice consumption. They differed in 4 respects: the current sample had higher BMI $(P=.035)$, higher socioeconomic status $(P=.03)$, more advanced pubertal status $(P=.04)$, and had mothers who reported lower levels of alcohol consumption $(P=.04)$ than the rest of the sample at 8 years of age. With regard to the initial cohort there were no differences (all $P \geq .07$ ) in BMI, mother's age at birth, maternal licorice consumption, gestation age, length at birth, and birthweight, but differed in that their mothers reported lower levels of alcohol consumption $(P=.04)$.

Sleep duration and quality were objectively measured using actigraphs (Actiwatch AW4 and AW7, CamNtech Ltd, Cambridge, United Kingdom). During the sleep registration period some of the participants took part in a very low dose overnight dexamethasone suppression test ${ }^{21}$; the night after dexamethasone intake at bedtime was excluded from sleep analysis, and serum samples were not collected at this time. Sleep was measured for an average of 7 nights at 8 years of age (SD, 1.2; range, 3-14) and 8 nights at 12 years of age (SD, 1.8; range 3-10). The measurement periods included both weekday nights ( 8 years of age: mean, 5.1 nights [SD, 1.0; range, 1-10]; 12 years of age: mean, 5.9 nights [SD, 1.5; range, 1-9]) and weekend nights ( 8 years of age: mean, 2 nights [SD, 0.4; range, 1-4]; 12 years of age: mean, 2 nights [SD, 1.0; range, $0-4]$ ).

Sleep duration refers to actual sleep time and was determined by the actigraph algorithm. Wake after sleep onset (WASO) time was defined as the amount of minutes when actual sleep time is subtracted from the assumed sleep. Sleep efficiency was defined as the ratio between actual sleep time and time in bed. Irregular sleep patterns were operationalized as the amount of catch-up sleep (calculated as the subtraction of weekday nights' sleep duration from weekend nights' sleep duration) during the weekend. Longer catch-up sleep is assumed to be an indicator of cumulative sleep debt from weekdays. The scoring was carried out as reported previously. ${ }^{19}$ Additionally, change variables were calculated as the subtraction of sleep variables at 12 years of age from 8 years of age, resulting in units that directly reflect the size of decrease of either sleep duration, or sleep quality as measured by WASO over time: the larger the change in duration, the more sleep duration decreases, and, the greater the change in WASO the more sleep quality improves.

Blood samples were collected at a clinical visit between 8:00 a.m. and 10:00 a.m. after an overnight fast. Serum total cholesterol (TC), high-density lipoprotein cholesterol (HDL-C) and LDL-C, and triglycerides (TGs) were analyzed in the clinical laboratory of Helsinki University Hospital.

Covariates measured in conjunction with sleep measurements and the clinical visit at 12 years of age include age, BMI 
Table I. Characteristics of the participants at 8 and 12 years of age

\begin{tabular}{|c|c|c|c|}
\hline & Girls* & Boys* & \\
\hline & Mean (SD) or $\mathbf{n}(\%)$ & Mean (SD) or $\mathbf{n}(\%)$ & $P$ \\
\hline \multicolumn{4}{|l|}{ Background characteristics } \\
\hline Age at first examination (8 y) & $8.1(0.32)$ & $8.1(0.31)$ & .94 \\
\hline Age at second examination (12 y) & $12.4(0.54)$ & $12.3(0.52)$ & .95 \\
\hline Time difference between 8 and 12 y of age (years) & $4.22(0.71)$ & $4.18(0.68)$ & .78 \\
\hline Parental education (12 y) & & & .17 \\
\hline Secondary or lower & $10(9.9 \%)$ & $11(12.4 \%)$ & \\
\hline Lower level tertiary & $30(29.7 \%)$ & $16(18 \%)$ & \\
\hline Upper level tertiary & $61(60.4 \%)$ & $62(69.6 \%)$ & \\
\hline BMI $(12 \mathrm{y})$ & $20.17(3.31)$ & $19.67(3.59)$ & .32 \\
\hline Physical activity $(12 \mathrm{y})^{\dagger}$ & $404.83(109.91)$ & $437.74(122.46)$ & .06 \\
\hline Pubertal development $(12 \mathrm{y})^{\ddagger}$ & $2.21(0.57)$ & $1.56(0.37)$ & $<.001$ \\
\hline \multicolumn{4}{|l|}{ Sleep characteristics } \\
\hline Sleep duration (h) (8 y) & $8.57(0.58)$ & $8.17(0.64)$ & .001 \\
\hline Sleep duration (h) (12 y) & $8.06(0.46)$ & $7.87(0.53)$ & .01 \\
\hline WASO (min) $(8 \mathrm{y})$ & $67.60(31.24)$ & 73.35 (27.76) & .33 \\
\hline WASO (min) (12 y) & $56.81(17.35)$ & $60.08(21.61)$ & .25 \\
\hline Sleep efficiency (\%) (8 y) & $85.83(5.53)$ & $83.69(5.08)$ & .04 \\
\hline Sleep efficiency (\%) (12 y) & $85.93(4.01)$ & $84.29(4.66)$ & .01 \\
\hline Catch-up sleep (h) (8 y) & $-.05(0.68)$ & $.03(0.56)$ & .48 \\
\hline Catch-up sleep (h) (12 y) & $0.36(0.78)$ & $0.22(0.80)$ & .14 \\
\hline \multicolumn{4}{|l|}{ Lipid profiles ${ }^{\S}$} \\
\hline $\mathrm{TC}(\mathrm{mmol} / \mathrm{L})(12 \mathrm{y})$ & $4.21(0.70)$ & $4.33(0.75)$ & .26 \\
\hline $\mathrm{HDL}-\mathrm{C}(\mathrm{mmol} / \mathrm{L})(12 \mathrm{y})$ & $1.58(0.32)$ & $1.70(0.37)$ & .03 \\
\hline LDL-C (mmol/L) (12 y) & $2.39(0.64)$ & $2.47(0.71)$ & .49 \\
\hline TGs (mmol/L) (12 y) & $0.92(0.54)$ & $0.70(0.34)$ & .001 \\
\hline
\end{tabular}

$P$ refers to the difference between girls and boys.

*The analytical sample consisted of 101 girls and 89 boys in cross-sectional analyses at 12 years of age, and 57 girls and 48 boys in longitudinal analyses. †Counts per minute.

†Pubertal Development Scale (1-4).

$\S$ Means and SDs are presented as arithmetic values, log-transformed variables were used for further analyses.

$\left(\mathrm{kg} / \mathrm{m}^{2}\right)$, Pubertal Development Scale, physical activity, and socioeconomic status. The Pubertal Development Scale is a validated scale ${ }^{22}$ of pubertal maturation. For girls, the selfreported scale had 5-items: body hair, growth spurt, skin changes, menarche, and breast development. For boys, the 3 first items were similar, and additionally facial hair and voice change were rated. All the items were scored on a scale of no changes yet (1) to clear changes (3). Following Mustanski et al, ${ }^{23}$ we omitted the fourth option (development is complete) in all other scales except menarche $(1=$ no, $4=$ has occurred $)$ because of the young age distribution of our sample.

Physical activity was measured using omnidirectional accelerometers (Actiwatch AW7) and by calculating the daily activity count mean based on 1-minute intervals. The watch was worn on the nondominant wrist for a minimum of 4 days at 12 years of age and data were analyzed as described previously by Martikainen et al. ${ }^{21}$ Socioeconomic status was parent reported when the child was 12 years of age and defined as highest achieved education of either parent. Education was classified as secondary or lower, lower level tertiary, or upper level tertiary.

\section{Statistical Analyses}

We used IBM SPSS Statistics 23.0 (IBM Corp, Armonk, New York) for all statistical analyses. Our main predictor variables were standardized scores of sleep duration, WASO, and, weekend catch-up sleep at ages 8 and 12 years of age, and the arithmetic difference, change, between these variables over the 4-year period.
Lipid variables (HDL-C, LDL-C, TC, TGs) were transformed logarithmically to normalize skewed distributions, and then standardized to mean of 0 and standard deviation of 1 . Linear regression analyses tested associations between sleep variables and lipids. We ran the analyses separately in girls and boys, because boys lag behind girls in pubertal development and because there are mean-level differences between girls and boys in sleep and lipid variables. We made adjustments for age (model 1) and thereafter for BMI, pubertal development, physical activity, and socioeconomic status (model 2).

\section{Results}

Table I presents sample characteristics by sex. As reported previously, at 8 years of age in comparison with girls, boys had shorter sleep duration, ${ }^{19}$ at 12 years of age boys lagged behind girls in pubertal development, ${ }^{19}$ had shorter sleep duration, ${ }^{24}$ lower sleep efficiency, ${ }^{19}$ higher HDL-C, and lower TGs. At 8 years of age, $53 \%$ of girls and $50 \%$ of boys slept more during weekends than weekdays. At 12 years of age, $74 \%$ of girls and $66 \%$ of boys slept more during weekends than weekdays. According to the Finnish growth reference, the BMI cutoff points for overweight and obesity at 12.3 years of age are 22.5 and 28.1 for girls and 21.0 and 25.5 for boys, respectively. ${ }^{25}$ Based on these criteria, $84 \%$ of girls, and $72 \%$ of boys in our sample are within normal weight range.

Sleep duration (Pearson $r=0.35$ and $0.39[P<.001]$ for girls and boys, respectively), WASO (Pearson $r=0.59$ and 0.39 


\begin{tabular}{|c|c|c|c|c|c|c|c|c|c|c|c|c|}
\hline \multirow[b]{2}{*}{ Girls } & \multicolumn{3}{|c|}{ TC } & \multicolumn{3}{|c|}{ LDL-C } & \multicolumn{3}{|c|}{ HDL-C } & \multicolumn{3}{|c|}{ TGs } \\
\hline & B & (95\% Cl) & $P$ & B & (95\% Cl) & $P$ & B & (95\% Cl) & $P$ & B & (95\% Cl) & $P$ \\
\hline \multicolumn{13}{|c|}{ Sleep at 8 years of age } \\
\hline \multicolumn{13}{|c|}{ Duration } \\
\hline Model 1 & -0.16 & $(-0.49,0.17)$ & .33 & -0.15 & $(-0.48,0.18)$ & .36 & 0.32 & $(0.03,0.62)$ & .03 & -0.39 & $(-0.69,-.09)$ & .01 \\
\hline Model 2 & -0.12 & $(-0.48,0.23)$ & .49 & -0.12 & $(-0.48,0.23)$ & .49 & 0.31 & $(0.01,0.62)$ & .04 & -0.36 & $(-0.68,-.04)$ & .03 \\
\hline \multicolumn{13}{|l|}{ WASO } \\
\hline Model 1 & 0.20 & $(-0.10,0.51)$ & .19 & 0.08 & $(-0.23,0.39)$ & .61 & -0.12 & $(-0.41,0.17)$ & .40 & 0.34 & $(.06,0.63)$ & .02 \\
\hline Model 2 & 0.22 & $(-0.12,0.55)$ & .20 & 0.09 & $(-0.26,0.43)$ & .62 & -0.12 & $(-0.42,0.18)$ & .43 & 0.33 & $(.02,0.64)$ & .04 \\
\hline \multicolumn{13}{|c|}{ Sleep efficiency } \\
\hline Model 1 & -0.20 & $(-0.52,0.11)$ & .20 & -0.11 & $(-0.43,0.22)$ & .51 & 0.17 & $(-0.12,0.47)$ & .25 & -0.39 & $(-0.68,-0.10)$ & .01 \\
\hline Model 2 & -0.21 & $(-0.57,0.14)$ & .23 & -0.11 & $(-0.47,0.25)$ & .53 & 0.19 & $(-0.13,0.50)$ & .24 & -0.39 & $(-0.71,-0.06)$ & .02 \\
\hline \multicolumn{13}{|c|}{ Catch-up sleep } \\
\hline Model 1 & 0.20 & $(-.06,0.46)$ & .13 & 0.10 & $(-0.16,0.37)$ & .43 & 0.22 & $(-0.03,0.46)$ & .08 & -0.17 & $(-0.42,0.08)$ & .18 \\
\hline Model 2 & 0.25 & $(-.04,0.53)$ & .09 & 0.14 & $(-0.16,0.43)$ & .36 & 0.24 & $(-0.02,0.50)$ & .07 & -0.17 & $(-0.45,0.11)$ & .24 \\
\hline \multicolumn{13}{|c|}{ Sleep at 12 years of age } \\
\hline \multicolumn{13}{|c|}{ Duration } \\
\hline Model 1 & .04 & $(-0.16,0.25)$ & .68 & 0.03 & $(-0.18,0.23)$ & .79 & 0.22 & $(0.01,0.42)$ & .04 & -0.19 & $(-0.40,0.02)$ & .08 \\
\hline Model 2 & -.02 & $(-0.24,0.21)$ & .89 & 0.00 & $(-0.22,0.22)$ & .99 & 0.18 & $(-0.04,0.39)$ & 11 & -0.19 & $(-0.43,0.04)$ & .11 \\
\hline \multicolumn{13}{|l|}{ WASO } \\
\hline Model 1 & .01 & $(-0.18,0.20)$ & .94 & -0.09 & $(-0.28,0.10)$ & .34 & 0.02 & $(-0.18,0.21)$ & .87 & 0.10 & $(-0.09,0.30)$ & .30 \\
\hline Model 2 & .06 & $(-0.15,0.26)$ & .58 & -0.03 & $(-0.23,0.17)$ & .79 & -0.02 & $(-0.22,0.17)$ & .83 & 0.16 & $(-0.06,0.37)$ & 0.15 \\
\hline \multicolumn{13}{|c|}{ Sleep efficiency } \\
\hline Model 1 & .04 & $(-0.16,0.23)$ & .72 & 0.13 & $(-.06,0.32)$ & .18 & -0.02 & $(-0.23,0.18)$ & .82 & -0.06 & $(-0.27,0.14)$ & .53 \\
\hline Model 2 & .01 & $(-0.20,0.21)$ & .94 & 0.10 & $(-0.10,0.30)$ & .34 & -0.01 & $(-0.20,0.19)$ & .95 & -0.09 & $(-0.31,0.12)$ & .40 \\
\hline \multicolumn{13}{|c|}{ Catch-up sleep } \\
\hline Model 1 & 0.10 & $(-0.11,0.31)$ & .35 & 0.08 & $(-0.13,0.29)$ & .46 & -0.04 & $(-0.24,0.17)$ & .74 & 0.24 & $(0.03,0.46)$ & .02 \\
\hline Model 2 & 0.10 & $(-0.11,0.31)$ & .33 & 0.06 & $(-0.15,0.27)$ & .56 & 0.01 & $(-0.18,0.20)$ & .89 & 0.21 & $(0.00,0.42)$ & .05 \\
\hline
\end{tabular}

Model 1 is adjusted for age at 12 years of age. Model 2 is adjusted for age, BMI, physical activity, pubertal development, socioeconomic status (measured at 12 years of age).

$[P<.001]$ for girls and boys, respectively), and sleep efficiency (Pearson $r=0.59$ and $0.39[P<.001]$ for girls and boys, respectively) showed significant rank-order stability from 8 to 12 years of age. Catch-up sleep during weekends was not significantly correlated from age 8 to 12 years of age (Pearson $r=.05$ and $0.12[P>.27]$ for girls and boys, respectively).

Table II (available at www.jpeds.com) shows the correlations between the covariates and the dependent variables. In girls, higher BMI at 12 years of age correlated with lower HDL$\mathrm{C}$, and higher LDL-C and TGs (Pearson $r \geq 0.24 ; P \leq .01$ ). In boys, higher BMI at 12 years of age correlated with lower HDL$\mathrm{C}$, and higher TGs (Pearson $r \geq 0.24 ; P \leq .01$ ). A more advanced pubertal development correlated with lower LDL-C and TC (Pearson $r \geq 0.31 ; P \leq .001$ ) in boys. No other correlations were statistically significant.

Table III shows that, in girls, shorter sleep duration at 8 years of age was associated with lower HDL-C and higher TGs; higher WASO and lower sleep efficiency at 8 years of age was associated with higher TGs; and longer catch-up sleep during weekends at 12 years of age was associated with higher TGs (models 1 and 2). Shorter sleep duration at 12 years of age was also associated with lower HDL-C, but this association did not persist after adjustments for physical activity and parental education (model 2; $P>.05$ ).

Figure 1 illustrates that, in girls, a smaller decrease in sleep duration from 8 to 12 years of age was associated with higher TC and LDL-C. Also, in girls a larger decrease in WASO from 8 to 12 years of age was associated with higher TC (Figure 2).

In boys, only 2 associations were significant: longer sleep duration at 8 years of age was associated with higher TGs (model
2: standardized regression coefficient $\mathrm{B}=0.38$ [95\% CI, 0.08$0.69 ; P=.016]$; model $1: P>.05)$; and a larger decrease in sleep duration from age 8 to 12 years of age was associated with higher TGs in both models (Figure 1, C).

\section{Discussion}

There are 3 main findings in this study. First, in girls shorter sleep duration and poorer sleep quality in childhood was associated with a more atherogenic lipid profile in early adolescence, over a 4-year follow-up period. This shows how sleep and lipids are related longitudinally before full adolescence, regardless of adjustment for several confounders, such as physical activity level. Although cross-sectional associations were also detected, the longitudinal associations were even more profound. This finding is in line with previous cross-sectional studies. ${ }^{10,13}$

Our second finding showed that there are differences in associations between sleep and lipids in girls and boys. In girls, longer and better quality sleep was associated positively with cholesterol markers, and in boys significant associations were only found between sleep and TGs. Previous research regarding the sex-specific associations between sleep and metabolism and obesity is in line with our findings. ${ }^{11,13,14}$ The association we found between sleep duration at 8 years of age and TGs in boys became significant only in the adjusted model.

Third, change in sleep duration and quality during the transition from childhood to adolescence is also an important aspect of normative development, and, in the current study, it had a beneficial effect on lipid profiles in girls. At 12 years of age, 


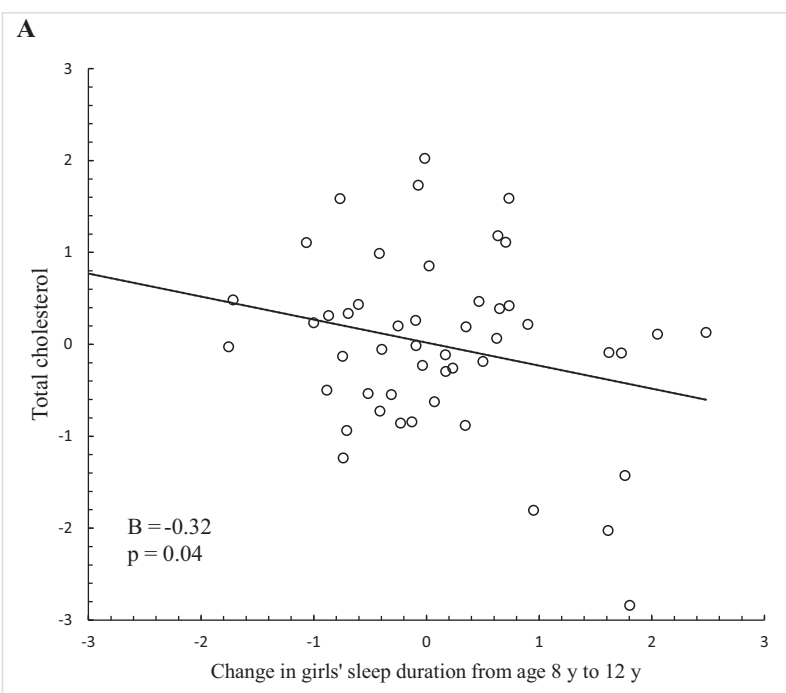

B

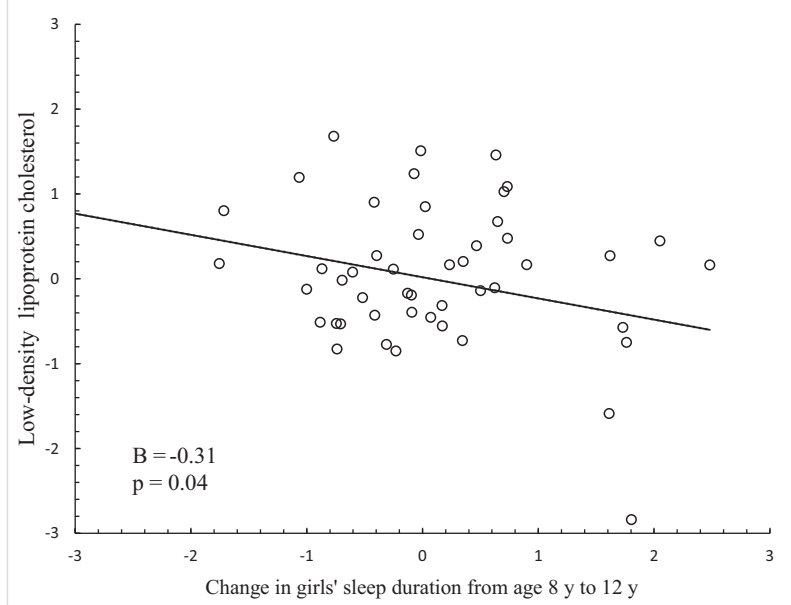

C

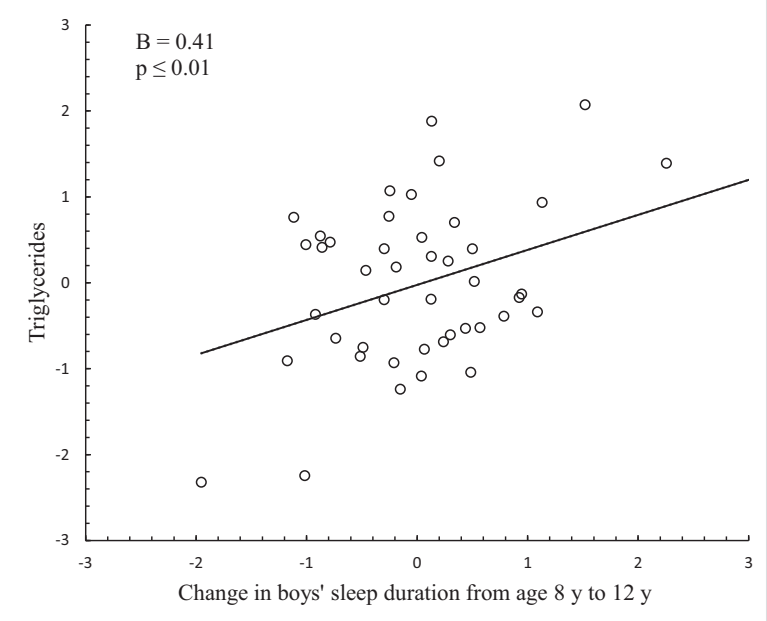

Figure 1. Associations between lipid markers and change in sleep duration as standardized units after controlling for age, pubertal development, BMI, socioeconomic status, and physical activity. Greater change indicates greater decrease in sleep duration. Line represents linear fit.

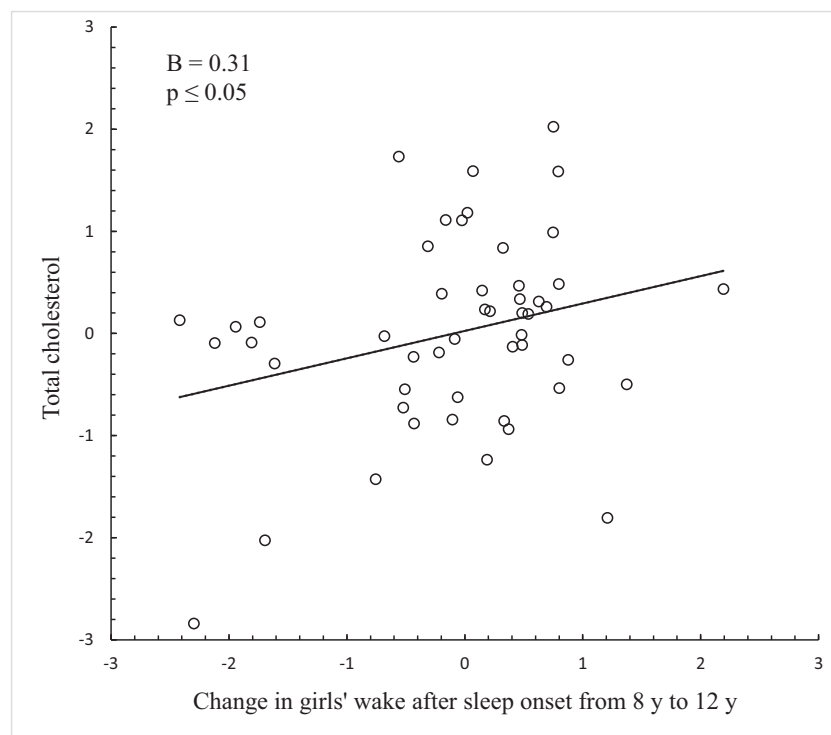

Figure 2. Association between TC and change in WASO in girls as standardized units after controlling for age, pubertal development, BMI, socioeconomic status, and physical activity. Greater change indicates greater decrease in WASO. Line represents linear fit.

boys' pubertal development is in its early stages whereas among girls it is more advanced. Although we have no explanation for our sex-specific findings, pubertal development may be one component. Given that sleep duration typically decreases during the transition from childhood to adolescence, and sleep quality as measured by sleep efficiency improves, ${ }^{19,26}$ changes toward these developmental directions in sleep were beneficial considering the lipid profile: a decrease in sleep duration was manifested in lower TC and lower LDL-C in girls. An improvement in sleep quality was associated with higher TC, but not with other lipid markers. However, in boys, the only significant associations between sleep and lipids were found in TGs, which are considered sensitive to food intake.

Additionally, we found that irregular sleep patterns measured by catch-up sleep in early adolescence (at 12 years of age) were associated with higher TGs in girls. Because TGs are highly sensitive to food intake, and insufficient sleep affects appetite, it is possible that cumulative weekday sleep debt, or great variance in sleep patterns, leads to increased appetite. Weekend catchup sleep was not associated with any lipid markers in boys. Previous studies have reported that weekend catch-up sleep may have some beneficial effects on metabolic outcomes, ${ }^{10,17}$ but in our study those associations were not found.

There is increasing evidence regarding the relationship between sleep and metabolic function. As previous research has suggested, sleep is associated with hormonal changes, including those in ghrelin, leptin, and insulin levels. ${ }^{27-29}$ A recent review reported metabolic and endocrine alterations, including decreased glucose tolerance, decreased insulin sensitivity, increased evening concentrations of cortisol, increased levels of ghrelin, and decreased levels of leptin having associations 
with insufficient sleep..$^{30}$ This "nocturnal programming" may promote the development of adverse lipid profiles and increase risk for diabetes.

One SD longer sleep duration (38.4 minutes) in girls at 8 years of age corresponded roughly with a 7\% increase in HDL-C and a $17 \%$ lower level of TGs. Fragmented sleep, as indicated by 1 SD higher WASO (32.8 minutes), at 8 years of age corresponded roughly with an $18 \%$ elevation in TGs. These associations may not be linear and there is evidence regarding the harms of very long sleep. ${ }^{31}$

The strength of our study was a rather long measurement period of sleep in a representative community cohort. The measurement periods extended over both weekday and weekend nights during typical, representative time periods over the school semester. Additionally, we were able to control for several potential confounders such as physical activity level.

As a limitation, we did not have lipid profile data from 8 years of age, restricting the potential to study the development of lipid profiles over time. This is a question for future research. Also as a limitation, the analytical sample in this study represented only a small subsample of the original cohort raising potential for selection bias.

This study suggests that even in populations with a healthy weight range, sleep has a long-term impact. This emphasizes the need for sufficient sleep in childhood.

Submitted for publication Feb 18, 2016; last revision received May 4, 2016; accepted Jun 8, 2016

Reprint requests: Liisa Kuula, MA, Institute of Behavioural Sciences, University of Helsinki, P.O. Box 9, Helsinki 00014, Finland. E-mail: liisa.kuula -paavola@ helsinki.fi

\section{References}

1. Cappuccio FP, Taggart FM, Kandala NB, Currie A, Peile E, Stranges S, et al. Meta-analysis of short sleep duration and obesity in children and adults. Sleep 2008;31:619-26.

2. Touchette E, Petit D, Tremblay RE, Boivin M, Falissard B, Genolini C, et al. Associations between sleep duration patterns and overweight/obesity at age 6. Sleep 2008;31:1507-14.

3. Gruber R, Cassoff J, Frenette S, Wiebe S, Carrier J. Impact of sleep extension and restriction on children's emotional lability and impulsivity. Pediatrics 2012;130:e1155-61.

4. Armstrong JM, Ruttle PL, Klein MH, Essex MJ, Benca RM. Associations of child insomnia, sleep movement, and their persistence with mental health symptoms in childhood and adolescence. Sleep 2014;37:901-9.

5. Raikkonen K, Matthews KA, Pesonen AK, Pyhala R, Paavonen EJ, Feldt $\mathrm{K}$, et al. Poor sleep and altered hypothalamic-pituitary-adrenocortical and sympatho-adrenal-medullary system activity in children. J Clin Endocrinol Metab 2010;95:2254-61.

6. Ruiz N, Rangel A, Rodriguez C, Rodriguez L, Rodriguez V. Relationship among nocturnal sleep deficit, excess weight and metabolic alterations in adolescents. Arch Argent Pediatr 2014;112:511-8.

7. Narang I, Manlhiot C, Davies-Shaw J, Gibson D, Chahal N, Stearne K, et al. Sleep disturbance and cardiovascular risk in adolescents. CMAJ 2012;184:E913-20.

8. Koren D, Levitt Katz LE, Brar PC, Gallagher PR, Berkowitz RI, Brooks LJ. Sleep architecture and glucose and insulin homeostasis in obese adolescents. Diabetes Care 2011;34:2442-7.

9. Iglayreger HB, Peterson MD, Liu D, Parker CA, Woolford SJ, Sallinen Gafka BJ, et al. Sleep duration predicts cardiometabolic risk in obese adolescents. J Pediatr 2014;164:1085-90. e1.
10. Spruyt K, Molfese DL, Gozal D. Sleep duration, sleep regularity, body weight, and metabolic homeostasis in school-aged children. Pediatrics 2011;127:e345-52.

11. Kong AP, Wing YK, Choi KC, Li AM, Ko GT, Ma RC, et al. Associations of sleep duration with obesity and serum lipid profile in children and adolescents. Sleep Med 2011;12:659-65.

12. Sung V, Beebe DW, Vandyke R, Fenchel MC, Crimmins NA, Kirk S, et al. Does sleep duration predict metabolic risk in obese adolescents attending tertiary services? A cross-sectional study. Sleep 2011;34:891-8.

13. Azadbakht L, Kelishadi R, Khodarahmi M, Qorbani M, Heshmat R, Motlagh ME, et al. The association of sleep duration and cardiometabolic risk factors in a national sample of children and adolescents: the CASPIAN III study. Nutrition 2013;29:1133-41.

14. Berentzen NE, Smit HA, Bekkers MB, Brunekreef B, Koppelman GH, De Jongste JC, et al. Time in bed, sleep quality and associations with cardiometabolic markers in children: the Prevention and Incidence of Asthma and Mite Allergy birth cohort study. J Sleep Res 2014;23:3-12.

15. Gangwisch JE, Malaspina D, Babiss LA, Opler MG, Posner K, Shen S, et al. Short sleep duration as a risk factor for hypercholesterolemia: analyses of the National Longitudinal Study of Adolescent Health. Sleep 2010;33:956-61.

16. Cespedes EM, Rifas-Shiman SL, Redline S, Gillman MW, Pena MM, Taveras EM. Longitudinal associations of sleep curtailment with metabolic risk in mid-childhood. Obesity (Silver Spring) 2014;22:2586-92.

17. Wing YK, Li SX, Li AM, Zhang J, Kong AP. The effect of weekend and holiday sleep compensation on childhood overweight and obesity. Pediatrics 2009;124:e994-1000.

18. Strandberg TE, Jarvenpaa AL, Vanhanen H, McKeigue PM. Birth outcome in relation to licorice consumption during pregnancy. Am J Epidemiol 2001;153:1085-8.

19. Pesonen AK, Martikainen S, Heinonen K, Wehkalampi K, Lahti J, Kajantie E, et al. Continuity and change in poor sleep from childhood to early adolescence. Sleep 2014;37:289-97.

20. Raikkonen K, Pesonen AK, Heinonen K, Lahti J, Komsi N, Eriksson JG, et al. Maternal licorice consumption and detrimental cognitive and psychiatric outcomes in children. Am J Epidemiol 2009;170:1137-46.

21. Martikainen S, Pesonen AK, Lahti J, Heinonen K, Pyhala R, Tammelin T, et al. Physical activity and hypothalamic-pituitary-adrenocortical axis function in adolescents. Psychoneuroendocrinology 2014;49:96-105.

22. Petersen AC. A self-report measure of pubertal status: reliability, validity, and initial norms. J Youth Adolesc 1988;17:117-33.

23. Mustanski BS, Viken RJ, Kaprio J, Pulkkinen L, Rose RJ. Genetic and environmental influences on pubertal development: longitudinal data from Finnish twins at ages 11 and 14. Dev Psychol 2004;40:1188-98.

24. Pesonen AK, Martikainen S, Kajantie E, Heinonen K, Wehkalampi K, Lahti $J$, et al. The associations between adolescent sleep, diurnal cortisol patterns and cortisol reactivity to dexamethasone suppression test. Psychoneuroendocrinology 2014;49:150-60.

25. Saari A, Sankilampi U, Hannila ML, Kiviniemi V, Kesseli K, Dunkel L. New Finnish growth references for children and adolescents aged 0 to 20 years: length/height-for-age, weight-for-length/height, and body mass indexfor-age. Ann Med 2011;43:235-48.

26. Williams JA, Zimmerman FJ, Bell JF. Norms and trends of sleep time among us children and adolescents. JAMA Pediatr 2013;167:55-60.

27. Taheri S, Lin L, Austin D, Young T, Mignot E. Short sleep duration is associated with reduced leptin, elevated ghrelin, and increased body mass index. PLoS Med 2004;1:e62.

28. Boeke CE, Storfer-Isser A, Redline S, Taveras EM. Childhood sleep duration and quality in relation to leptin concentration in two cohort studies. Sleep 2014;37:613-20.

29. Javaheri S, Storfer-Isser A, Rosen CL, Redline S. Association of short and long sleep durations with insulin sensitivity in adolescents. J Pediatr 2011;158:617-23.

30. Leproult R, Van Cauter E. Role of sleep and sleep loss in hormonal release and metabolism. Endocr Dev 2010;17:11-21.

31. Cappuccio FP, D’Elia L, Strazzullo P, Miller MA. Sleep duration and allcause mortality: a systematic review and meta-analysis of prospective studies. Sleep 2010;33:585-92. 


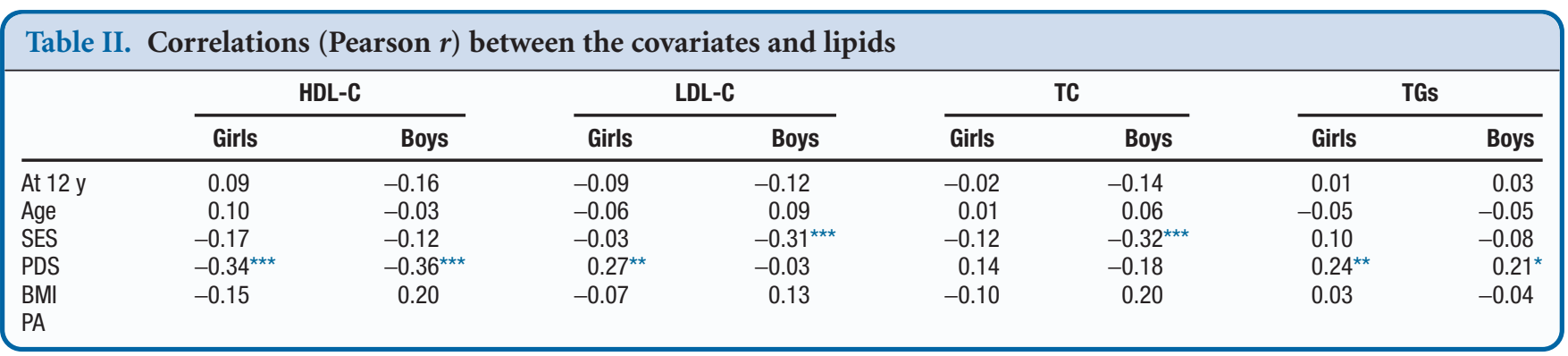

$P A$, physical activity, $P D S$, Pubertal Development Scale, SES, socioeconomic status.

${ }^{\star} P<.05$.

${ }^{\star \star} P<.01$.

${ }^{\star \star \star} P<.001$. 